\title{
Translation of Shaanxi Characteristic Dietary Names from the Perspective of Skopos Theory
}

\author{
Hui Liu ${ }^{1, *}$, Tingting Han $^{2, *}$ \\ ${ }^{1}$ School of Foreign Language, Xi'an Shiyou University, Xi'an, Shaanxi 710065, China \\ ${ }^{2}$ School of Foreign Lnaguage, Xi'an Shiyou University, Xi'an, Shaanxi 710065, China \\ *Corresponding author.Email: liuhui1020@xsyu.edu.cn,1109703638@qq.com
}

\begin{abstract}
Under the background of the strategy of "going global" of Chinese culture, the culture with local characteristics has gradually entered the world. Food culture is an important part of local characteristic culture. Based on the analysis of the connotation of Shaanxi characteristic dietary culture, this paper explores the translation techniques of Shaanxi characteristic dietary names under the guidance of Skopos theory. According to the features of Shaanxi characteristic dietary names, it puts forward the following skills: translating raw materials and cooking methods, adding raw materials or cooking information, and translating cultural information as well as transliteration, which can help Shaanxi culture go global.
\end{abstract}

Keywords: Skopos theory, Shaanxi characteristic diet, translation

\section{INTRODUCTION}

From ancient and modern times, people's life needs can always promote the social development to a certain extent. It is inevitable that the pursuit and needs of nobles and people in ancient Shaanxi for daily diet make this place become the earliest birthplace of food culture in China. Shaanxi' s catering culture has a long history with many historical stories and folk tales as well as profound cultural connotations. Nowadays, as the starting point of "one belt and one road", Shaanxi will play its due role in the national communication and create a glorious dietary culture. Therefore, an increasing number of translators begin to pay close attention to the translation of Shaanxi catering culture. Based on the Skopos Theory, this paper further explores the translation of Shaanxi characteristic dietary names.

\section{The Cultural Connotation of Shaanxi Food}

When exploring the translation of Shaanxi characteristic dietary names, we must understand what is Shaanxi's dietary culture. Similarly, if we want to understand the food culture of Shaanxi, we must know what is food culture. Some people think that the narrow sense of dietary culture particularly refers to an art involved in human beings' eating and drinking, and those think that the broad sense of dietary culture refers to the knowledge of how human beings (or a nation) eat[1]. In view of the understanding of the concept of "dietary culture", Wang Yingquan (2017) believes that dietary culture covers the whole process of dietary activities in human history and constitutes its own specific disciplinary field[2]. In view of the status of "food culture" in Chinese social and cultural construction, famous scholars have also expressed their views. Cai Yuanpei thinks cooking and eating belong to civilization, so regards them as "dietary culture". Qian Xuesen (1994), a great scholar, shows that diet is also a kind of culture and he says dietary culture has rich contents in Chinese historical tradition. Although scholars have different expressions, they all display the special status of dietary culture and its function in the construction of national culture at present. Therefore, the connotation of "dietary culture" has been enriched with the progress and development of the times, and its position in people's lives has been increased.

Scholars have different opinions on the connotation of Shaanxi's dietary culture. Wang Zihui (2014) believes that food culture in Shaanxi is as elegant and open-minded as people over there[3]. Li Xi (2013) believes that even the present Shaanxi home-cooked diet still has the lingering charm of Zhou, Qin, Han and Tang Dynasties. The "Wei" in Shaanxi Province has its own characteristics, which originate from the "Chun" and "Wo" in the "Eight Treasures of the Western Zhou Dynasty"[4]. The catering circles also thinks that "Lü Shi Chun Qiu” which is regarded as the highest achievement of Chinese Pre-Qin Cuisine, is nurtured and developed in Shaanxi. Thus, it can be seen that Shaanxi diet has its unique charm and profound cultural connotation. Therefore, it is particularly crucial to translate the names of Shaanxi characteristic diet in order to better show the world Shaanxi characteristic diet culture. This paper also explores the translation of the names of Shaanxi characteristic diet in the perspective of Skopos Theory.

\section{Translations in the Perspective of Skopos Theory}

Skopos theory is an important translation theory. It was originated from Germany in the 1970s. In 1971, Katharina 
Reiss, a German scholar, expressed her opinions that the special purpose which translation should be achieved can be regarded as a criterion for judging whether a translation was good or not, which laid a foundation for the establishment of functional translation theory. After that, the development of Skopos theory was continuously enriched and developed before it came into being. "Skopos" is a Latin vocabulary, meaning "purpose". Hans Vermeer (1984), the main representative of Skopos Theory, argues that translation methods and strategies must be determined by the purpose of the target text. Besides, he also put forward that Skopos rule, coherence rule and fidelity rule should be included in Skopos Theory[5]. The Skopos Principle determines the whole process of translation. That is, the whole process of translation must be guided by its purpose. The principle of coherence refers to the fact that the recipient can understand the translated information, and the translated text should play its functions in the cultural and communicative context of the target language. The rule of faithfulness refers to the interlingual coherence between the source text and the target text. That is, the target text must be faithful to the source text. The degree and form of faithfulness are also determined by the target text and the understanding of the target text. These three principles are complementary and play an important role in the translator's process of translation. In Vermeer's opinion, the principle of purpose is the highest guiding principle, and the principles of coherence and faithfulness give service to it.

\section{TRANSLATION OF SHAANXI CHARACTERISTIC DIETARY NAMES UNDER THE GUIDANCE OF SKOPOS THEORY}

Shaanxi characteristic diet has a great variety and is rich in cultural connotations. In Skopos theory, the purpose of translation determines the whole process of translation. When translating the names of Shaanxi characteristic diets, there are two main purposes. The first purpose is that the the target language can accurately introduce the raw materials and cooking methods of food. The second one is that translations can arouse the curiosity and interest of the target language readers for Shaanxi characteristic diets, so as to publicize the Shaanxi characteristic catering culture. Therefore, when translating the names of Shaanxi characteristic diets, translators should follow the principle of purpose as well as accurately convey the information of raw materials and cooking methods of Shaanxi characteristic diets to the target readers. At the same time, translators should follow the principle of coherence and faithfulness, as well as express accurately the legends and historical stories contained in Shaani characteristic diets so that the target language readers can better understand Shaanxi's characteristic diets to help Shaanxi culture "go out".

\subsection{Translating Raw Materials and Cooking Methods}

In Chinese diets, some characteristic diets are named after raw materials and cooking methods. In order to accurately convey the information of the raw materials and cooking methods of diets to the target language readers, we can translate raw materials and cooking methods of characteristic food directly. For example, the Chinese name of "Yan Xun $\mathrm{Ya} \mathrm{Zi}$ " contains raw materials and cooking methods, which can be translated into "Smoked duck". This translation not only contains raw materials of this diet, but also introduces the cooking methods of it. Thus, the target language readers can understand the cooking information of the diet more simply and directly, and achieve the purpose of transmitting accurately information including raw materials and cooking method. Therefore, this is a successful translation which translators can use in the processing of translating.

Example: Yang Rou Pao Mo

"Yang Rou Pao Mo" is formally named as "ancient soup". The method of making it is complicated. First, wash and cut high-quality beef and mutton; Second, prepare scallion, ginger and other condiments as well as boil them together in a pot; Finally, choose suitable soup. "Mo" is a kind of cake. People have to break it into small pieces and put them into a bowl, then ask cooks for help. When translating this characteristic diet, translators can translate its raw materials and cooking methods directly. It is translated as "Steamed mutton" from the very beginning. Although this translation adopts the method of translating directly raw methods and cooking methods, there is only one kind of raw materials involved without another essential information--Mo. Therefore, this translation method does not accurately and completely convey the information of raw materials and cooking methods to the target language readers, and can not achieve the purpose of publicizing the Shaanxi characteristic diets. The Chinese name of "Yang Rou Pao Mo" itself contains information about raw materials and cooking methods, which can be translated as "Pita Bread Soaked in Lamb Soup".

This translation not only contains all the raw materials of this diet, but also introduces the cooking methods so that the target language readers can understand the cooking information of it more simply and directly. It achieves the primary purpose of accurately conveying the information of the raw materials and cooking methods of the diet, and also achieves the ultimate goal of publicizing Shaanxi characteristic food.

\subsection{Adding Raw Materials or Cooking Information}

In some Shaanxi characteristic diets, their names include cooking tools or are named after cooking tools. During the process of translating such dietary names, translating directly cooking tools can not accurately convey the information of raw materials and cooking methods to the 
target language readers, which can not arrive translators' translation purpose. Therefore, translators should first translate raw materials or cooking methods and then add additional information like cooking methods.

Example: Guo Kui

“锅盔” is also called as pot helmet steamed bun or dry bun, which is a popular pasta snack in Guanzhong area of Shaanxi Province. It is branded with wheat flour making it more crisp. It is named "Guo Kui" because its shape is similar to the helmet worn by ancient soldiers. Therefore, translators can utilize methods mentioned above when translate it that translators can translate the raw materials or cooking method, then add the information of cooking methods. "Guo Kui" is translated as "Crispy Pastry" and "Crispy pancake" from the very beginning. Both of them emphasize that it is a kind of pastries and cakes, but target languages readers can not find counterparts in their local food. They can not understand what it is. Thus, these translations can not achieve the purpose that accurately convey dietary information to target languages readers. Wang Wenhao, an English professor at Sichuan University, once suggested that "Guo Kui" can be translated into "Pie". He said that there were something in a pie, which was the same as "Guo Kui". Therefore, the foreigners could find counterparts in their dietary culture. However, there are still some deviations in the expression and it can not express the characteristics of this food, which causes some misunderstandings to the target language readers. According to the principle of purpose, it should be translated into "Crusty pancake--a kind of large round baked wheat cake, popular in Shaanxi Province". This translation not only gives the raw materials of this food, but also adds the information of cooking methods, so that the target language readers can comprehensively and accurately understand the raw materials and the information of cooking methods of it, so as to achieve the purpose of attracting the target language readers and publicizing Shaanxi characteristic diets.

\section{3 .Translating Cultural Information}

Some characteristic diets in Shaani are named after folk tales and legends. Translating the name of these diets directly does not convey the information about the raw materials and cooking methods to the target language readers. Sometimes, it also makes the target language readers feel confused. Therefore, translator can choose to translate cultural information carried by diets in order to convey accurately the information of the raw materials and cooking methods to the target language readers as well as to enable the target language readers to better understand the cultural information contained by diets. For example, if "Fu Qi Fei Pian" is translated into "wife and husband's lungs", it not only fails to accurately convey the information about raw materials and cooking methods of the diet to the target languages readers, but also make them feel confused and horrified. The purpose of publicizing Shaanxi's characteristic diets can not be achieved.
According to the principle of coherence and faithfulness in Skopos theory, translators can translate "Fu Qi Fei Pian" into "Sliced Beef and Ox Tongue in Chilli Sauce". This translation contains raw materials and cooking methods of this diet, so that the target language readers can understand the cooking information more simply and directly, which can achieve the purpose of expressing accurately the cultural information carried by this diet and publicizing Shaanxi's characteristic diets. Translators can draw on this method mentioned above in the process of translating characteristic diets.

Example: Shi Zi Mo

"Shi Zi Mo" is made by baking cakes on hot stones. It can be translated into "Stone Steamed Bread" by directly translating its name. However, this translation fails to accurately convey the raw materials and cooking methods of the food to the target language readers as well as makes target language readers confused, which can not fulfill the purpose of publicizing Shaanxi's characteristic food. Therefore, according to the principle of coherence and faithfulness in Skopos theory, it can be translated into "Pancake Baked with Pebbles", "Crispy Pastry Baked on Hot Cobbles" and "The Steamed Bun Baked by Heated Rocks" in order to achieve the purpose of accurately conveying the information of the raw materials and cooking methods of the diet contained in it, so that the target language readers can understand the cooking information of the diet more simply and directly. It can arouse target language readers' interests, make them understand more about the cultural information carried by it as well as achieve the purpose of cultural publicity.

\subsection{Adopting Transliteration}

Translators often encounter some situations that they can not find the corresponding vocabularies and images of characteristic diets in target language. It is difficult for translators to accurately express the meanings of characteristic diets in one or two words. Under this condition, it is necessary to translate directly the pronunciation of the original text into the same or similar counterparts in the target language, which can make the translation concise and succinct as well we achieve the purpose of publicizing dietary culture. For example, "Hun Tun" can be translated into "wonton". This kind of translation is concise and vivid as well as can also achieve the purpose of cultural publicity. Translators can adopt such a method during the process of translating Shaanxi characteristic diets.

Example: Guan Zhong Jian Bing

Pancakes are characteristic diets in Guanzhong. The making method is exquisite and demanding. Excellent quality of wheat flour should be prepared. Then, water is added into flour to make it to paste. After that, condiments are sprinkled into the paste and it can be branded. "Guan Zhong Jian Bing" is translated into "Pancake" from the very beginning. However, this translation is undesirable, because the making method of it is different from that of ordinary pancakes. Pancakes are usually baked, while this 
characteristic diet is branded. Therefore, this translation can not accurately express the characteristics of this diet and can not achieve the purpose of publicizing Shaanxi's characteristic diets. Because there is no corresponding vocabularies and images of this characteristic diet in target language, translators can adopt the method that expresses its translation with the same or similar speech sounds in the source language. Thus, "Guan Zhong Jian Bing" can be translated into Jianbing in Guanzhong Style" or "Guanzhong Pancake". This kind of translation is quite succinct and is easily understood and mastered by the target language readers. Therefore, it can arouse target language readers' interests in Shaanxi catering culture and achieve the translation purpose of publicizing Shaanxi characteristic catering culture.

\section{SUMMARY}

To sum up, the translation of local characteristic dietary names from the perspective of Skopos Theory should follow the principles of purpose, coherence and faithfulness. In the process of translation, translators should adopt different translation skills according to various kinds of characteristic dietary names, such as translating raw materials and cooking methods, adding raw materials or cooking information, and translating cultural information as well as transliteration, in order to attract target language readers and publicize Shaanxi catering culture. Successful translations of dietary names can help the culture of Shaanxi go out and strengthen the soft power of Shaanxi culture.

\section{ACKNOWLEDGMENT}

This research was financially supported by the research project "On Standardization of Shaanxi Cultural Term Translation in the Light of 'Going Global' Strategy" (2016K030) funded by Shaanxi provincial social science foundation; and the graduate students' research project of innovation and practice of Xi'an Shiyou University ( YCS 19141012).

\section{REFERENCES}

[1] Yibo Guan. Comparison of Chinese and Western food culture[J]. Journal of Hubei Open Vocational College, 2011, 24(04): 90-91.

[2] Yingquan Wang. On the Formation and Development of Shaanxi Food Culture--Taking Shaanxi Cuisine as an Example [J]. Xi'an: Journal of Shannxi Institute of Socialism, 2017(3): 54-56.

[3] Zihui Wang. On the Dietary Culture of the
Guanzhong Areas [M]. Xi'an: Xi'an Publishing House: 2014.

[4] Xi Li. On Shaanxi Food Culture [M]. Xi'an: Shaanxi Normal University Publishing Co., Ltd., 2013.

[5] Peter Newmark. Approaches to Translation [M]. Shanghai: Shanghai Foreign Language Education Press, 2001. 